\title{
Osteosarcoma periosteal desdiferenciado en la diáfisis del cúbito
}

\author{
Miguel Ángel Clara-Altamirano ${ }^{1, *}$, Dorian Yarih García-Ortega ${ }^{2}$, Alejandro Maciel-Miranda $^{3}$, \\ Héctor Martínez-Said ${ }^{2}$, Jorge Luis Martínez-Tlahuel ${ }^{4}$, Claudia Haydee Sarai Caro-Sánchez ${ }^{5}$ \\ y Mario Cuellar-Hubbe ${ }^{6}$
}

\begin{abstract}
${ }^{1}$ Ortopedista Oncólogo adscrito al Departamento de Piel y Partes Blandas; ${ }^{2}$ Cirujano Oncólogo adscrito al Servicio de Piel y Partes Blandas; ${ }^{3}$ Cirujano Plático adscrito al Servicio de Piel y Partes Blandas; ${ }^{4}$ Servicio de oncología Medica; ${ }^{5}$ Patóloga Oncóloga adscrita al Servicio de Patología Quirúrgica. ${ }^{6}$ Cirujano Oncólogo, Jefe de Servicio de Piel y Partes blandas. Instituto Nacional de Cancerología, Ciudad de México, México.
\end{abstract}

Recibido el 4 de junio de 2016; aceptado el 25 de febrero de 2017

Disponible en Internet el 4 de agosto de 2017

\section{PALABRAS CLAVE \\ Osteosarcoma \\ parosteal \\ desdiferenciado; \\ Resección intercalar; \\ Injerto autólogo \\ microvascularizado}

\begin{abstract}
Resumen Introducción: El osteosarcoma parosteal desdiferenciado es una variante en la cuali un osteosarcoma de alto grado coexiste con un osteosarcoma parosteal. Caso clínico: Paciente de sexo femenino, de 20 años de edad, con 6 meses de evolución de dolor y limitación funcional en antebrazo derecho, sin causa aparente. Se le realizan radiografías y se observa una lesión tumoral en la diáfisis del cúbito derecho. A la exploración física presenta dolor a la palpación y limitación a la pronosupinación. La tomografía computada de tórax muestra enfer medad metastásica en el lóbulo superior del pulmón izquierdo. Se le realiza biopsia incisional de tumoración en el cúbito derecho, con reporte de osteosarcoma parosteal desdiferenciado, por lo que inicia manejo con quimioterapia neoadyuvante con cisplatino y doxorubicina hasta completar tres ciclos. El tratamiento quirúrgico consistió en la resección intercalar de la diáfisis del cúbito derecho más reconstrucción con injerto autólogo microvascularizado de diáfisis de peroné derecho y estabilización del injerto con placa DCP $3.5 \mathrm{~mm}$ y placa de tercio de caña. En el mismo procedimiento se le realiza metastasectomía pulmonar por toracoscopía. Reporte histopatológico posquirúrgico con necrosis del $100 \%$. Actualmente la paciente se encuentra asintomática, sin datos de actividad tumoral. Conclusión: El osteosarcoma parosteal desdi ferenciado es una patología rara, pero que se debe sospechar como diagnóstico diferenciak̂ ante un osteosarcoma parosteal. Se debe tener en cuenta que esta enfermedad puede generar metástasis por su patrón desdiferenciado. Es importante planificar un tratamiento quirúrgico que permita una adecuada reconstrucción funcional, siempre teniendo en cuenta el principio oncológico. (creativecommons.org/licenses/by-nc-nd/4.0/).
\end{abstract}

*E-mail para correspondencia: drmiguelclara@gmail.com (M.A. Clara-Altamirano) 


\section{INTRODUCCIÓN}

Los osteosarcomas de superficie comprenden un grupo variado de tumores óseos malignos con diferente grado de malignidad ${ }^{1,2}$. Dentro de este grupo, el osteosarcoma parosteal desdiferenciado es una variante en la cual un osteosarcoma de alto grado coexiste con un osteosarcoma parosteal o de bajo grado, ya sea al mismo tiempo (forma sincrónica) o como recurrencia (forma metacrónica) $)^{2-4}$.

El osteosarcoma paraosteal típico es una lesión superficial formada por fibroblastos de bajo grado que producen el hueso laminar $^{5}$. Se presenta habitualmente entre los 20 y los 40 años de edad, siendo su localización más frecuente la región posterior del fémur distal ${ }^{1,6}$.

En la literatura existen pocos casos publicados de osteosarcoma parosteal desdiferenciado. Los hallazgos clinicopatológicos, el diagnóstico, el tratamiento y la evolución de los pacientes con este raro osteosarcoma no han sido bien definidos ${ }^{4 \cdot 6}$.

\section{CASO CLÍNICO}

Paciente de sexo femenino, de 20 años de edad, con cuadro clínico de 6 meses de evolución, con dolor en el antebrazo derecho que le limita la movilidad, sin causa aparente. A la exploración física presenta dolor a la palpación en la diáfisis del cúbito derecho y limitación a la pronosupinación por

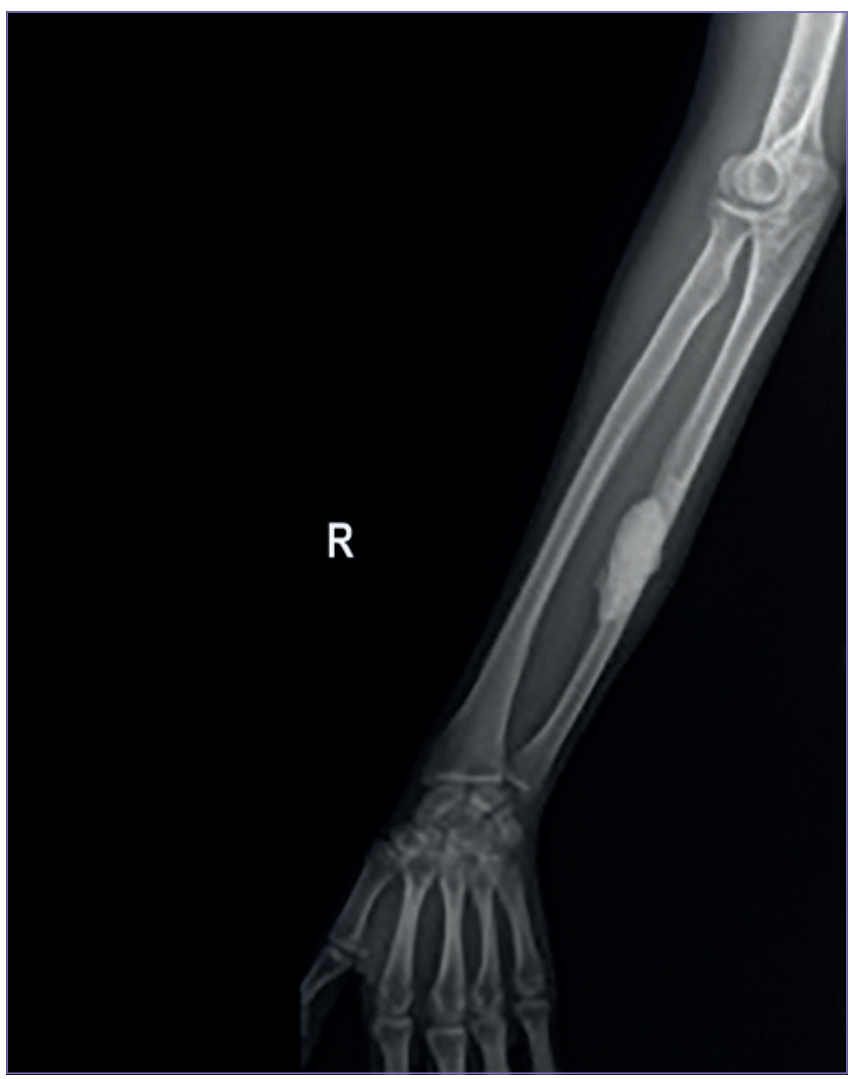

Figura 1. Radiografía anteroposterior del antebrazo derecho en la que se observa una imagen radioopaca en el tercio medio de la diáfisis del cúbito, con extensión a partes blandas, compatible con un osteosarcoma parosteal.

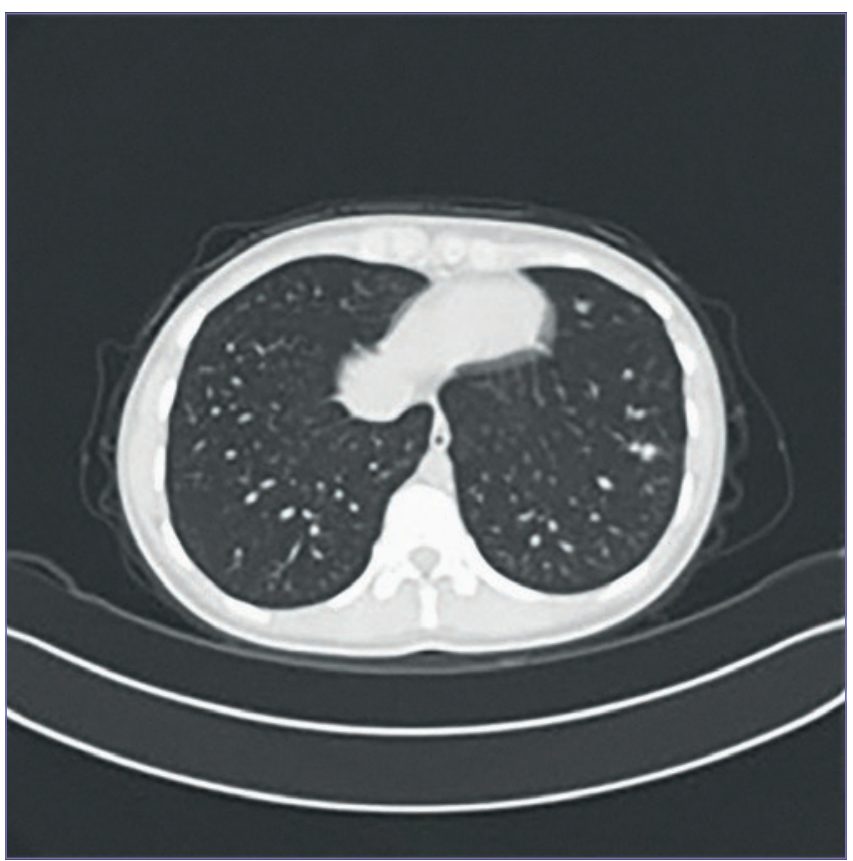

Figura 2. Tomografía computada de tórax, con presencia de lesión metastásica en el lóbulo superior del pulmón izquierdo.

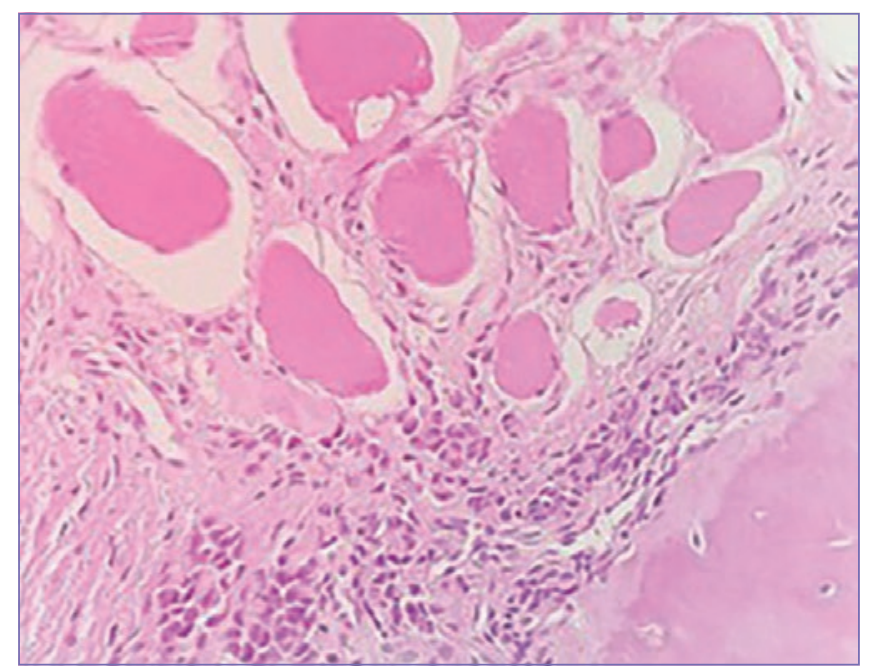

Figura 3. Biopsia (40x). Se identifican hueso compacto en la esquina inferior derecha de la imagen y osteoblastos activos con atipia en la periferia que infiltran el tejido muscular adyacente, así como depósito de material osteoide entre estas.

dolor. Se le solicitan radiografías del antebrazo (Fig. 1), en las que se observa una tumoración en el tercio medio de la diáfisis del cúbito derecho, dependiente de la cortical, con extensión a partes blandas, radiológicamente compatible con un osteosarcoma parosteal.

La tomografía computada de tórax muestra múltiples nódulos pulmonares en probable relación con depósitos secun-darios de primario conocido, el de mayor tamaño localizado en la periferia de la base del pulmón izquierdo, que mide $8 \mathrm{~mm}$ (Fig. 2). Se realiza biopsia incisional de la tumoración en el tercio medio de la diáfisis de cúbito, con reporte histopatológico de osteosarcoma parosteal desdiferenciado, Enneking III, AJCC IV A (Fig. 3). 


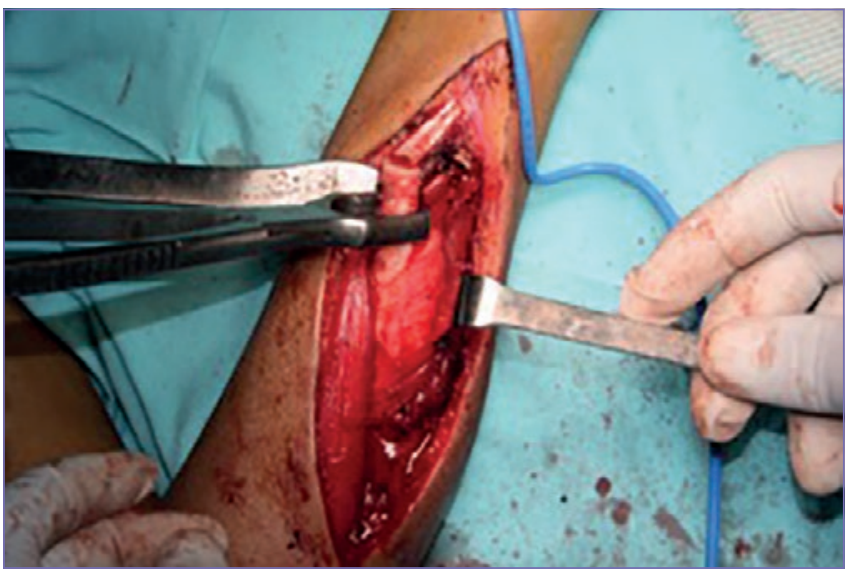

Figura 4. Resección intercalar de la diáfisis del cúbito derecho.

Inicia quimioterapia neoadyuvante con cisplatino y doxorubicina hasta completar tres ciclos. Posterior a la quimioterapia neoadyuvante, se realiza resección intercalar de diáfisis de cúbito derecho más reconstrucción con injerto autólogo microvascularizado de diáfisis de peroné derecho, y estabilización del injerto mediante colocación de placa DCP $3.5 \mathrm{~mm}$ y de placa de tercio de caña (Figs. 4 y 5). Posteriormente se le realiza, por parte del servicio de cirugía de tórax, metastasectomía pulmonar mediante resección pulmonar en cuña por toracoscopía, con reporte histopatológico de márgenes de resección negativos y necrosis del $100 \%$ (Huvos grado IV), con producto de metastasectomía sin evidencia de células neoplásicas viables (respuesta completa). Actualmente, a los 12 meses de seguimiento, la paciente se encuentra asintomática, sin datos de actividad tumoral local ni a distancia, tolerando la movilización del antebrazo y de la mano (Fig. 6).

\section{DISCUSIÓN}

El osteosarcoma parosteal desdiferenciado es una afección rara, pero que debe sospecharse como diagnóstico diferencial ante un osteosarcoma parosteal; además, se debe tener en cuenta que esta enfermedad puede generar metástasis por su patrón desdiferenciado.

La desdiferenciación en el osteosarcoma parosteal es reportada frecuentemente en las recurrencias del mismo. Wold, et al. ${ }^{7}$ describieron 11 casos de desdiferenciación del osteosarcoma parosteal. En diez de estos casos, la desdiferenciación se documentó en la primera, segunda o tercera recurrencia, mientras que la coexistencia de zonas de bajo y alto grado fue reportada en un solo caso.

El caso que presentamos ilustra la importancia de considerar la posibilidad de la coexistencia de áreas desdiferenciadas en el osteosarcoma parosteal, así como la posibilidad de que estas zonas puedan generar metástasis.

Diversos autores señalan que debe considerarse al osteosarcoma parosteal desdiferenciado como diferente del osteosarcoma de superficie de alto grado, ya que la evolución clínica y la mortalidad son diferentes, con una menor tasa de mortalidad para el osteosarcoma desdiferenciado ${ }^{6,8,9}$.

Se considera la invasión al canal medular como un factor pronóstico para la sobrevida global y el periodo libre

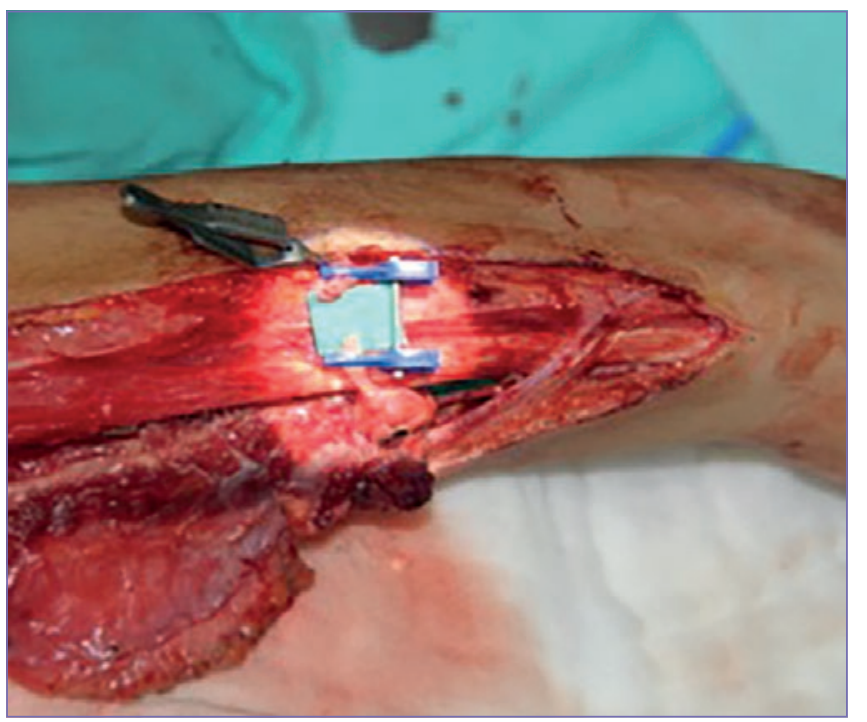

Figura 5. Anastomosis vascular del injerto de peroné vascularizado a la arteria cubital.

de enfermedad en aquellos pacientes con osteosarcoma desdiferenciado ${ }^{6,10}$. En la actualidad se reportan rangos de sobrevida del $80 \%$ en pacientes con osteosarcoma parosteal desdiferenciado asociando quimioterapia neoadyuvante más tratamiento quirúrgico con márgenes amplios ${ }^{5,6,10}$.

Existe una fuerte correlación entre el pronóstico y el volumen de necrosis tumoral en los pacientes tratados con quimioterapia neoadyuvante y cirugía; este porcentaje se evalúa mediante la clasificación de Huvos ${ }^{11}$. En este caso se observó una respuesta excelente (necrosis del 100\%) poste-

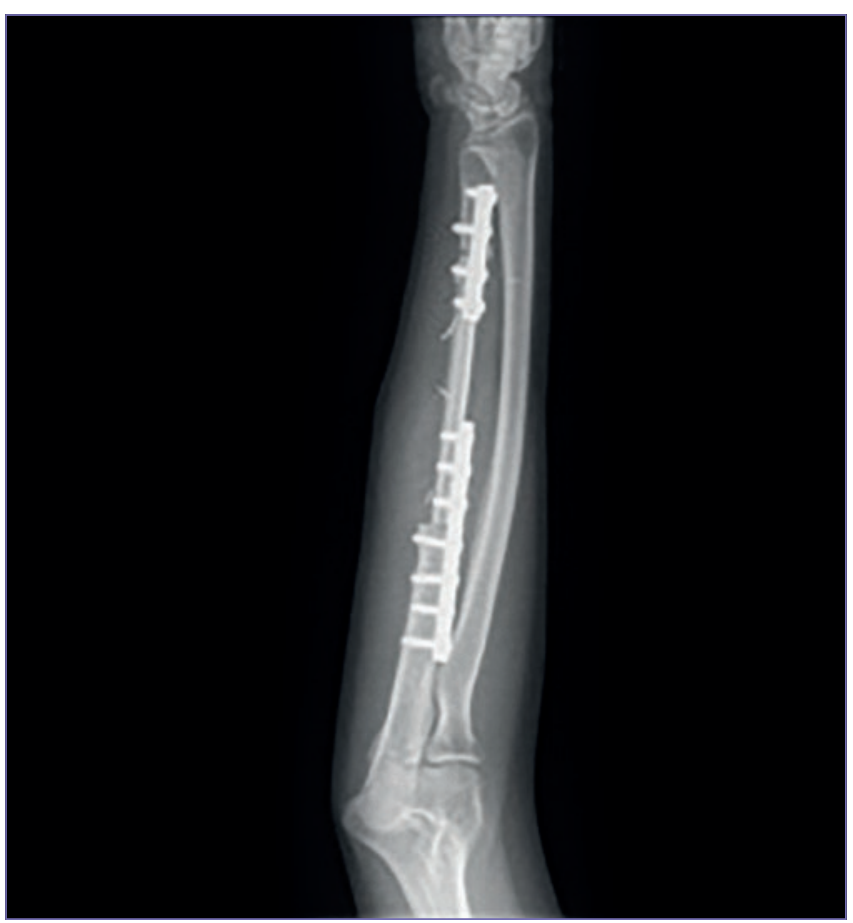

Figura 6. Control radiográfico posquirúrgico en el que se observa la adecuada colocación del material de osteosíntesis y del injerto de peroné vascularizado. 
rior al tratamiento neoadyuvante. Además, es importante considerar que la reducción del tamaño del tumor como respuesta a la quimioterapia neoadyuvante hace que la lesión sea mucho más fácil de resecar.

La reconstrucción con injerto autólogo de peroné vascularizado permite una mejor y más rápida integración del injerto en la zona receptora, sin ocasionar limitación funcional en la zona donante ${ }^{12}$. Taylor, et al. ${ }^{13}$ reportaron la primera transferencia de peroné libre vascularizado en 1975.

Weiland, et al. ${ }^{14-18}$ han descrito el uso de injertos de hueso vascularizados para el tratamiento de varias patologías, como resección de tumores, defectos de hueso postraumáticos y pseudoartrosis congénita. Se ha tratado de definir el papel de esta técnica en la reconstrucción de tumores de huesos largos ${ }^{19}$, y se ha propuesto que pudiera estar indicada en defectos mayores de $6 \mathrm{~cm}$ tras resecciones tumorales. Esta técnica permite la transferencia de hasta $24 \mathrm{~cm}$ de hueso, con un pedículo vascular de $8 \mathrm{~cm}$ en promedio ${ }^{20}$.

En conclusión, el osteosarcoma parosteal desdiferenciado es un osteosarcoma localizado predominantemente en la superficie ósea, en el cual se encuentran un componente parosteal de bajo grado asociado con un componente sarcomatoso de alto grado. El comportamiento del osteosarcoma parosteal desdiferenciado es más agresivo que el del osteosarcoma parosteal convencional, y debe sospecharse la presencia de enfermedad metastásica. Es importante planificar un tratamiento quirúrgico que permita una adecuada reconstrucción funcional, siempre teniendo en cuenta el principio oncológico.

\section{BIBLIOGRAFÍA}

1. Petrilli AS, de Camargo B, Filho VO, et al. Results of the Brazilian Osteosarcoma Treatment Group Studies III and IV: prognostic factors and impact on survival. J Clin Oncol. 2006;24:1161.

2. Bacci G, Longhi A, Versari M, et al. Prognostic factors for osteosarcoma of the extremity treated with neoadjuvant chemotherapy: 15-year experience in 789 patients treated at a single institution. Cancer. 2006;106:1154.
3. Arslan HS, Aydin O, Dervıșoğlu S, et al. Low grade osteosarcoma, review of 15 cases in a series of 156 osteosarcoma cases. Turk Patoloji Derg. 2011;27:138-43.

4. Bertoni F, Bacchini P, Staals EL, et al. Dedifferentiated parosteal osteosarcoma: the experience of the Rizzoli institute. Cancer. 2005;103:2373-82.

5. Van Der Spuy DJ. Osteosarcoma: pathology, staging and management. SA Orthopaedic Journal. 2009;69-78.

6. Schwab JH, Antonescu CR, Athanasian EA, et al. A comparison of intramedullary and juxtacortical low-grade osteogenic sarcoma. Clin Orthop Relat Res. 2008;466:1318-22.

7. Wold LE, Unni KK, Beabout JW, et al. Dedifferentiated parosteal os teosarcoma. J Bone Joint Surg Am. 1984;66:53-9.

8. Van Oven MW, Molenaar WM, Freling NJM. Dedifferentiated parosteal osteosarcoma of the femur with aneuploidy and lung metastases. Cancer. 1989;63:807-11.

9. Okada K, Frassica FJ, Sim FH, et al. Parosteal osteosarcoma. A clinicopathological study. J Bone Joint Surg Am. 1994;76A:366-78.

10. Sheth DS, Yasko AW, Raymond AK, et al. Conventional and dedifferentiated parosteal osteosarcoma. Diagnosis, treatment and outcome. Cancer. 1996;78:2136-45.

11. Huvos AG. Bone tumors: diagnosis, treatment, and prognosis. 2nd ed. London, U.K.: W.B. Saunders; 1991.

12. Zelenski N, Brigman BE, Levin LS, et al. The vascularized fibular graft in the pediatric upper extremity: a durable, biological solution to large oncologic defects. Sarcoma. 2013;2013:321201.

13. Taylor GI, Miller GD, Ham FJ. The free vascularized bone graft. $\bar{A}$ clinical extension of microvascular techniques. Plast Reconstr Surg. 1975;55:533-44.

14. Weiland AJ, Moore JR, Daniel RK. Vascularized bone autografts: experience with 41 cases. Clin Orthop. 1983;174:87.

15. Weiland AJ. Current concepts review: vascularized free bone trans plants. J Bone Joint Surg Am. 1981;63A:166.

16. Weiland AJ, Daniel RK, Riley LH. Application of the free vascularized bone graft in the treatment of malignant or aggressive bone tumors. Johns Hopkins Med J. 1977;140:85.

17. Weiland AJ, Daniel RK. Microvascular anastomosis of bone graft in the treatment of massive defects in bone. J Bone Joint Surg Am? 1979;61A:98.

18. Weiland AJ, Daniel RK. Congenital pseudoarthrosis of the tibia: treat ment with vascularized autogenous fibula graft - a preliminary report. Johns Hopkins Med J. 1980;147:89.

19. Gao YH, Ketch LL, Eladoumikdachi F, et al. Upper limb salvage with microvascular bone transfer for major long-bone segmental tumor resections. Ann Plast Surg. 2001;47:240-6.

20. Murray PM. Free vascularized bone transfer in limb salvage surgery of the upper extremity. Hand Clin. 2004;20:203-11. 